\title{
Planar graphs have two-coloring number at most $8^{*}$
}

\author{
Zdeněk Dvořák ${ }^{\dagger} \quad$ Adam Kabela Tomáš Kaiser $^{\ddagger}$
}

\begin{abstract}
We prove that the two-coloring number of any planar graph is at most 8. This resolves a question of Kierstead et al. [SIAM J. Discrete Math. 23 (2009), 1548-1560]. The result is optimal.
\end{abstract}

\section{Introduction}

We study the two-coloring number of graphs. This parameter was introduced by Chen and Schelp [2] under the name of $p$-arrangeability; they related it to the Ramsey numbers of graphs and the Burr-Erdôs conjecture [1]. It was subsequently found to be related to coloring properties of graphs, such as the game chromatic number, the acyclic chromatic number or the degenerate chromatic number (see [3] and the references therein).

We now recall the definition of the two-coloring number. Let $G$ be a graph and let $\prec$ be a linear ordering of its vertices. (In this paper, graphs are allowed to have parallel edges, but not loops.) For a vertex $v \in V(G)$, let $L_{G, \prec}(v)$ be the set consisting of the vertices $u \in V(G)$ such that $u \prec v$ and either

- $u v \in E(G)$, or

\footnotetext{
${ }^{*}$ Supported by project GA14-19503S (Graph Colouring and Structure) of the Czech Science Foundation.

${ }^{\dagger}$ Computer Science Institute, Charles University, Prague, Czech Republic. E-mail: rakdver@iuuk.mff.cuni.cz.

${ }^{\ddagger}$ Department of Mathematics, University of West Bohemia, Pilsen, Czech Republic. E-mail: kabela@ntis.zcu.cz.

${ }^{\S}$ Department of Mathematics, Institute for Theoretical Computer Science (CE-ITI), and European Centre of Excellence NTIS (New Technologies for the Information Society), University of West Bohemia, Pilsen, Czech Republic. E-mail: kaisert@kma.zcu.cz.
} 
- $u$ and $v$ have a common neighbor $w \in V(G)$ such that $v \prec w$.

We say that an ordering $\prec$ is $d$-two-degenerate if $\left|L_{G, \prec}(v)\right| \leq d$ for every $v \in V(G)$. The two-coloring number $\operatorname{col}_{2}(G)$ of $G$ is defined as $d+1$ for the smallest integer $d$ such that there exists a $d$-two-degenerate ordering of the vertices of $G$.

Already in [2], the two-coloring number of planar graphs was bounded by an absolute constant, namely 761. The bound was improved to 10 in [4] and eventually to 9 in [3]. On the other hand, a planar graph with two-coloring number equal to 8 was constructed in [4]. Kierstead et al. [3] found simpler examples yielding the same lower bound (namely, any 5-connected triangulation in which the degree 5 vertices are non-adjacent has this property) and asked whether the two-coloring number of all planar graphs is bounded by 8.

We answer this question in the affirmative:

Theorem 1. The two-coloring number of any planar graph is at most 8 .

It was observed in [3] that the list-degenerate chromatic number of a graph $G$ is bounded by the two-coloring number of $G$. By this observation, Theorem 1 improves the known upper bound for the list-degenerate chromatic number of planar graphs, as well as for the ordinary degenerate chromatic number of planar graphs, to 8 .

The structure of this paper is as follows. In the remainder of this section, we formulate a more general version of Theorem 1 that is better suited for an inductive proof (Theorem 2 below). Section 2 focuses on the basic structural properties of a hypothetical minimal counterexample. These properties are used in Section 3 in a discharging procedure that provides a contradiction, establishing Theorem 2 and hence also Theorem 1 .

It will be useful to consider the following relative version of the notion of $d$-two-degenerate ordering. Let $G$ be a graph, let $C$ be a subset of its vertices and let $\prec$ be a linear ordering of $V(G) \backslash C$. For a vertex $v \in V(G) \backslash C$, let $L_{G, C, \prec}(v)$ be the set consisting of the vertices $u \in V(G) \backslash C$ such that $u \prec v$ and either

- $u v \in E(G)$, or

- $u$ and $v$ have a common neighbor $w \in V(G) \backslash C$ such that $v \prec w$, or

- $u$ and $v$ have a common neighbor $w \in C$.

We say that an ordering $\prec$ is $d$-two-degenerate relative to $C$ if $\left|L_{G, C, \prec}(v)\right| \leq d$ for every $v \in V(G) \backslash C$. 
Theorem 2. Let $G$ be a plane graph and let $K$ be a set of at most three vertices incident with the outer face of $G$. Let $C$ be a subset of $V(G)$ disjoint from $K$ such that every vertex of $C$ has at most 4 neighbors in $V(G) \backslash C$. There exists an ordering $\prec$ of $V(G) \backslash C$ that is 7 -two-degenerate relative to $C$, such that $u \prec v$ for every $u \in K$ and $v \in V(G) \backslash(C \cup K)$.

Note that Theorem 1 follows from Theorem 2 by setting $C=K=\emptyset$.

\section{Basic properties of a minimal counterexample}

Before we embark on the study of the properties of a minimal counterexample to Theorem 2, let us define the notion of minimality more precisely.

A target is a triple $(G, K, C)$, where $G$ is a plane graph, $K$ is the set of all vertices incident with the outer face of $G, 2 \leq|K| \leq 3$, and $C$ is a subset of $V(G)$ disjoint from $K$ such that every vertex of $C$ has at most 4 neighbors in $V(G) \backslash C$. Note that it suffices to show that Theorem 2 holds for every target, since if $|K| \leq 1$, then we can add $2-|K|$ new isolated vertices into the outer face of $G$ and include them in $K$, and we can add edges between the vertices of $K$ to ensure that the outer face of $G$ is only incident with the vertices of $K$. An ordering $\prec$ of $V(G) \backslash C$ is valid if $\prec$ is 7-two-degenerate relative to $C$ and $u \prec v$ for every $u \in K$ and $v \in V(G) \backslash(C \cup K)$. We say that a target $(G, K, C)$ is a counterexample if there exists no valid ordering $\prec$ of $V(G) \backslash C$. Let $s(G, K, C)=\left(n,-c, e_{C}, q,-t, e\right)$, where $n=|V(G)|$, $c=|C|, e_{C}$ is the number of edges of $G$ with at least one end in $C, q$ is the number of components of $G, t$ is the number of triangular faces of $G$, and $e=|E(G)|$. A target $\left(G^{\prime}, K^{\prime}, C^{\prime}\right)$ is smaller than $(G, K, C)$ if $s\left(G^{\prime}, K^{\prime}, C^{\prime}\right)$ is lexicographically smaller than $s(G, K, C)$ (observe that this establishes a well-quasiordering on targets). We say that a counterexample is minimal if there exists no smaller counterexample.

In a series of lemmas, we now establish the basic properties of minimal counterexamples.

Lemma 3. If $(G, K, C)$ is a minimal counterexample, then the following hold:

(i) $C$ is an independent set,

(ii) all vertices of $C$ have degree 4 ,

(iii) $G$ is connected, and

(iv) all faces of $G$ except possibly for the outer face have length 3. 
Proof. We prove claim (i). If an edge $e \in E(G)$ has both ends in $C$, then $(G-e, K, C)$ is a target smaller than $(G, K, C)$, and by the minimality of $(G, K, C)$, there exists a valid ordering $\prec$ for the target $(G-e, K, C)$. Note that $L_{G, C, \prec}(v)=L_{G-e, C, \prec}(v)$ for every $v \in V(G) \backslash C$, and thus $\prec$ is also valid for the target $(G, K, C)$, which is a contradiction. Hence, $C$ is an independent set in $G$.

We continue with claim (iii). Suppose that $G$ is not connected. Hence, $G$ contains a face $f$ incident with at least two distinct components $G_{1}$ and $G_{2}$ of $G$. If $G_{1}$ or $G_{2}$ consists of only one vertex $v \in C$, then $(G-v, K, C \backslash\{v\})$ is a target smaller than $(G, K, C)$ and its valid ordering is also valid for $(G, K, C)$, which is a contradiction. Otherwise, since $C$ is an independent set, there exist vertices $v_{1} \in V\left(G_{1}\right) \backslash C$ and $v_{2} \in V\left(G_{2}\right) \backslash C$ incident with $f$. Then, $\left(G+v_{1} v_{2}, K, C\right)$ is a target smaller than $(G, K, C)$ (with fewer components) and its valid ordering is also valid for $(G, K, C)$, which is a contradiction. Hence, $G$ is connected.

We next prove claim (iv). Suppose that $G$ has a non-outer face $f$ of length other than three. If $f$ has length 2 and not all its edges belong to the boundary of the outer face, then removing one of its edges results in a target smaller than $(G, K, C)$ whose valid ordering is also valid for $(G, K, C)$, which is a contradiction. If $f$ has length two and all its edges belong to the boundary of the outer face, then $V(G)=K$ and $(G, K, C)$ has a valid ordering, which is a contradiction. Hence, $f$ has length at least 4 . Let $f=v_{1} v_{2} v_{3} v_{4} \ldots$, with the labels chosen so that $v_{2} \in C$ if any vertex of $C$ is incident with $f$. Since $C$ is an independent set, it follows that $v_{1}, v_{3} \notin C$. If $v_{1} \neq v_{3}$, then $\left(G+v_{1} v_{3}, K, C\right)$ is a target smaller than $(G, K, C)$ (with more triangular faces) and its valid ordering is also valid for $(G, K, C)$, which is a contradiction. Hence, $v_{1}=v_{3}$.

If $v_{2} \in C$ and $v_{2}$ has degree at least two, then removing one of at least two edges between $v_{2}$ and $v_{1}=v_{3}$ results in a target smaller than $(G, K, C)$ whose valid ordering is also valid for $(G, K, C)$. If $v_{2} \in C$ and $v_{2}$ has degree exactly one, then $\left(G-v_{2}, K, C \backslash\left\{v_{2}\right\}\right)$ is a target smaller than $(G, K, C)$ whose valid ordering is also valid for $(G, K, C)$. In both cases, we obtain a contradiction, and thus $v_{2} \notin C$.

By the choice of the labels of $f$, it follows that no vertex of $C$ is incident with $f$. Furthermore, note that $v_{1}=v_{3}$ is a cut in $G$, and thus $v_{2} \neq v_{4}$. Consequently, $\left(G+v_{2} v_{4}, K, C\right)$ is a target smaller than $(G, K, C)$ whose valid ordering is also valid for $(G, K, C)$. This contradiction shows that every non-outer face of $G$ has length three.

It remains to prove claim (ii). Suppose that a vertex $v \in C$ has degree at most three. Since $v \notin K$, the faces incident with $v$ have length three, and 
thus the neighborhood of $v$ forms a clique in $G$. The target $(G-v, K, C \backslash\{v\})$ is smaller than $(G, K, C)$, and thus it has a valid ordering $\prec$. Suppose that for some vertices $x, y \in V(G) \backslash C$, we have $x \in L_{G, C, \prec}(y)$. If $v$ is not a common neighbor of $x$ and $y$, then clearly $x \in L_{G-v, C \backslash\{v\}, \prec}(y)$. If $v$ is a common neighbor of $x$ and $y$, then $x$ and $y$ are adjacent, and thus $x \in L_{G-v, C \backslash\{v\}, \prec}(y)$. It follows that $L_{G, C, \prec}(y)=L_{G-v, C \backslash\{v\}, \prec}(y)$ for every $y \in V(G) \backslash C$, and thus $\prec$ is a valid ordering for $(G, K, C)$. This is a contradiction, and thus all vertices of $C$ have degree at least 4 .

Note that a vertex of $C$ is not incident with parallel edges, as suppressing them would result in a target smaller than $(G, K, C)$ whose valid ordering is also valid for $(G, K, C)$. Since $C$ is an independent set and every vertex of $C$ has at most 4 neighbors not in $C$, it follows that all vertices in $C$ have degree exactly 4.

Consider a target $(G, K, C)$. For a vertex $v \in V(G) \backslash C$, let $a(v)$ be the number of neighbors of $v$ in $V(G) \backslash C$, and let $b(v)$ be the number of neighbors of $v$ in $C$ (counted with multiplicity when $v$ is incident with parallel edges). For non-negative integers $a, b$, we say that $v$ is an $(a, b)$-vertex if $a(v)=a$ and $b(v)=b$. Similarly, we say that $v$ is an $\left(a, \leq b^{\prime}\right)$-vertex if $a(v)=a$ and $b(v) \leq b^{\prime}$.

Corollary 4. If $(G, K, C)$ is a minimal counterexample and $v \in V(G) \backslash C$, then $a(v) \geq b(v)$. Furthermore, if $a(v)=b(v)$ and $u \in V(G) \backslash C$ is a neighbor of $v$, then $b(u) \geq 2$.

Proof. If $v \notin K$, then all faces incident with $v$ are triangles. If $v \in K$, then all faces except possibly for the outer one are triangles, and no vertex of the outer face belongs to $C$. Since $C$ is an independent set, at most half of the neighbors of $v$ belong to $C$, and thus $b(v) \leq a(v)$. Furthermore, if $b(v)=a(v)$, then every second neighbor of $v$ belongs to $C$, and thus $u$ and $v$ have two common neighbors belonging to $C$.

Lemma 5. If $(G, K, C)$ is a minimal counterexample and $v \in V(G) \backslash(K \cup$ $C)$, then $a(v) \geq 4$, and if $a(v)=4$, then $b(v)=4$.

Proof. Suppose for a contradiction that $v \in V(G) \backslash(K \cup C)$ satisfies either $a(v) \leq 3$, or $a(v)=4$ and $b(v) \leq 3$. By Corollary 4 , in the former case we have $b(v) \leq a(v)$.

Since $v$ has at most 4 neighbors in $V(G) \backslash C$, it follows that $(G, K, C \cup\{v\})$ is a target. Note that $(G, K, C \cup\{v\})$ is smaller than $(G, K, C)$, and let $\prec$ be its valid ordering. Extend $\prec$ to $V(G) \backslash C$ by letting $u \prec v$ for every

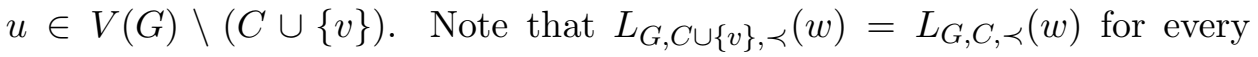




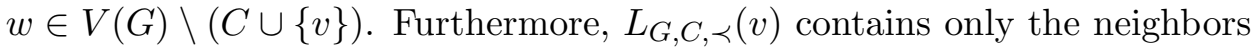
of $v$ that do not belong to $C$, and the vertices $z$ such that $z$ and $v$ have a common neighbor $w \in C$. However, since all faces of $G$ incident with $v$ are triangles and all vertices in $C$ have degree 4 , each neighbor $w \in C$ of $v$ has at most one neighbor $z$ not adjacent to $v$. Therefore, $\left|L_{G, C, \prec}(v)\right| \leq$ $\operatorname{deg}(v)=a(v)+b(v) \leq 7$, and thus $\prec$ is a valid ordering for $(G, K, C)$. This is a contradiction.

Lemma 6. Suppose that $(G, K, C)$ is a minimal counterexample. If $|K|=3$, then $G$ contains no parallel edges and all triangles in $G$ bound a face. If $|K|=2$, then the edges bounding the outer face of $G$ are the only parallel edges in $G$, and every non-facial triangle in $G$ contains a vertex of $C$ and both vertices of $K$.

Proof. Consider either a pair of parallel edges that do not bound the outer face of $G$, or a non-facial triangle in $G$. Since all faces of $G$ except for the outer one have length three, in the former case $G$ contains a non-facial cycle of length two. Hence, let $Q$ be a non-facial cycle of length 2 or 3 in $G$.

Suppose first that $V(Q) \cap C=\emptyset$. Let $G_{1}$ be the subgraph of $G$ drawn in the closure of the outer face of $Q$, and let $G_{2}$ be the subgraph of $G$ drawn in the closure of the inner face of $Q$. Let $C_{1}=C \cap V\left(G_{1}\right)$ and $C_{2}=$ $C \cap V\left(G_{2}\right)$. Note that $\left(G_{1}, K, C_{1}\right)$ and $\left(G_{2}, V(Q), C_{2}\right)$ are targets, and since $Q$ is a non-facial cycle, they are both smaller than $(G, K, C)$ and they have valid orderings $\prec_{1}$ and $\prec_{2}$, respectively. Let $\prec$ be the ordering of $V(G) \backslash C$ such that $u \prec v$ if $u, v \in V\left(G_{1}\right)$ and $u \prec_{1} v$, or if $u, v \in V\left(G_{2}\right) \backslash V(Q)$ and $u \prec_{2} v$, or if $u \in V\left(G_{1}\right)$ and $v \in V\left(G_{2}\right) \backslash V(Q)$.

Observe that for any $v \in V\left(G_{1}\right) \backslash\left(V(Q) \cup C_{1}\right)$, we have $L_{G, C, \prec}(v)=$ $L_{G_{1}, C_{1}, \prec_{1}}(v)$, since $v$ has no neighbors in $V\left(G_{2}\right)$ other than those belonging to $Q$ (which are also contained in $G_{1}$ ), and since $v \prec w$ for every $w \in V\left(G_{2}\right) \backslash$ $V(Q)$. Similarly, for any $v \in V\left(G_{2}\right) \backslash\left(V(Q) \cup C_{2}\right)$, we have $L_{G, C, \prec}(v)=$ $L_{G_{2}, C_{2}, \prec 2}(v)$, since $v$ has no neighbors in $V\left(G_{1}\right)$ other than those belonging to $Q$, and all the vertices of $Q$ are contained in $G_{2}$ and are smaller than $v$ in both orderings $\prec$ and $\prec_{2}$. Finally, for $v \in V(Q)$ we have $L_{G, C, \prec}(v)=$ $L_{G_{1}, C_{1}, \prec_{1}}(v)$, since all vertices of $V\left(G_{2}\right) \backslash\left(V(Q) \cup C_{2}\right)$ are greater than $v$ in $\prec$ and $Q$ is a clique, so all vertices of $Q$ smaller than $v$ in $\prec$ or $\prec_{1}$ belong to both $L_{G, C, \prec}(v)$ and $L_{G_{1}, C_{1}, \prec 1}(v)$. Furthermore, since $K \subseteq V\left(G_{1}\right)$, the choice of $\prec$ ensures that $u \prec v$ for every $u \in K$ and $v \in V(G) \backslash(C \cup K)$. Hence, $\prec$ is a valid ordering of $(G, K, C)$, which is a contradiction.

Therefore, every non-facial $(\leq 3)$-cycle in $G$ intersects $C$. Since $C$ is an independent set, $Q$ contains exactly one vertex of $C$. If $Q$ has length two, 
then removing one of the parallel edges of $Q$ results in a target smaller than $(G, K, C)$ whose valid ordering is also valid for $(G, K, C)$. It follows that $G$ contains no parallel edges except possibly for those bounding its outer face, and in particular $Q$ is a triangle.

Let $Q=v_{1} v_{2} v_{3}$, where $v_{1} \in C$. Let $e$ and $e^{\prime}$ be the edges of $G$ incident with $v_{1}$ distinct from $v_{1} v_{2}$ and $v_{1} v_{3}$. If exactly one of the edges $e$ and $e^{\prime}$ is contained in the open disk bounded by $Q$, then consider the neighbor $v_{4}$ of $v_{1}$ in the open disk bounded by $Q$. Since all faces incident with $v_{1}$ have length three, $v_{4}$ is adjacent to $v_{2}$ and $v_{3}$. Since the triangle $v_{2} v_{3} v_{4}$ does not intersect $C$, it bounds a face. However, this implies that $v_{4}$ is a $(2,1)$-vertex, which contradicts Lemma 5 .

If neither $e$ nor $e^{\prime}$ is contained in the open disk bounded by $Q$, then since $Q$ is not a facial triangle and all faces incident with $v_{1}$ have length three, it follows that $v_{2}$ and $v_{3}$ would be joined by a parallel edge drawn inside the open disk bounded by $Q$; however, this is impossible, since such a parallel edge is not incident with the outer face of $G$. Finally, consider the case that both $e$ and $e^{\prime}$ are contained in the open disk bounded by $Q$. Similarly, $v_{2}$ and $v_{3}$ are joined by a parallel edge, and thus $K=\left\{v_{2}, v_{3}\right\}$. We conclude that every non-facial triangle in $G$ contains a vertex of $C$ and two vertices of $K$.

Corollary 7. If $(G, K, C)$ is a minimal counterexample, then every vertex of $K$ has degree at least 4 .

Proof. Suppose first that a vertex $v \in K$ has degree two. Since all faces of $G$ except for the outer one are triangles, if $|K|=2$, this would imply that $G$ contains a loop, which is a contradiction. If $|K|=3$, then since all faces of $G$ are triangles and $G$ does not contain parallel edges, we have $V(G)=K$, and any ordering of $V(G)$ is valid, which is a contradiction.

Next, suppose that $v$ has degree three, and let $x$ be the neighbor of $v$ not belonging to $K$. If $|K|=2$, then since all faces incident with $x$ are triangles and $x$ is not incident with a parallel edge, it follows that $V(G)=K \cup\{x\}$ and $x$ has degree two. If $|K|=3$, say $K=\left\{v, y_{1}, y_{2}\right\}$, then since all faces of $G$ are triangles, it follows that $v x y_{1}$ and $v x y_{2}$ are triangles. Also, every triangle in $G$ is facial, and thus $x$ has degree three. In both cases, $x \notin C$ and $x$ is a $(2,0)$-vertex or a $(3,0)$-vertex, which contradicts Lemma 5 .

Let $\prec$ be an ordering of $V(G) \backslash C$ in a target $(G, K, C)$. For adjacent vertices $u \in V(G) \backslash C$ and $v$, a vertex $w \in V(G) \backslash C$ distinct from $u$ is a friend of $u$ via $v$ if $w \prec u$ and 
- $w=v$, or

- $v w \in E(G), u w \notin E(G)$, and $v \in C$, or

- $v w \in E(G), u w \notin E(G), u$ and $w$ do not have a common neighbor in $C$, and $u \prec v$.

Note that $L_{G, C, \prec}(u)$ consists exactly of the friends of $u$ via its neighbors. We will frequently use the following observations.

Lemma 8. Let $(G, K, C)$ be a minimal counterexample and let $u \in V(G) \backslash$ $(C \cup K)$ and $v \in V(G)$ be neighbors. Let $\prec$ be an ordering of $V(G) \backslash C$. Then the following hold:

(i) if $v \prec u$ or $v \in C$, then $u$ has at most one friend via $v$,

(ii) if $v \notin C \cup K$ and $u \prec v$, then $u$ has at most $a(v)-3$ friends via $v$,

(iii) if $v \notin C \cup K, u \prec v$, and $v$ has a neighbor $r \notin C$ non-adjacent to $u$ such that $u \prec r$ and no vertex of $C$ is a common neighbor of $u, v$ and $r$, then $u$ has at most $a(v)-4$ friends via $v$.

Proof. (i) If $v \prec u$, then $v$ is the only friend of $u$ via $v$. If $v \in C$, then since all faces incident with $v$ are triangles and $v$ has degree 4, the vertex $v$ has at most one neighbor not adjacent to $u$, and thus $u$ has at most one friend via $v$.

(ii) Suppose that $v \notin C \cup K$ and $u \prec v$. By Lemma 5, we have $a(v) \geq 4$, and since all faces incident with $v$ have length three, it follows that $v$ has at least two neighbors $z_{1}, z_{2} \notin C$ distinct from $u$ such that for $i \in\{1,2\}$, either $u v z_{i}$ is a face, or $z_{i}$ and $u$ have a common neighbor $z_{i}^{\prime} \in C$ such that $u v z_{i}^{\prime}$ is a face. In either case, $z_{i}$ is not a friend of $u$ via $v$. Since $u$ is not a friend of $u$ via $v$, it follows that $u$ has at most $a(v)-3$ friends via $v$.

(iii) Let us now additionally assume that $v$ has a neighbor $r$ as described in the last case of the lemma. Using the notation from the previous case, we first show that the vertex $z_{1}$ is distinct from $r$. This is clearly the case if $z_{1}$ is adjacent to $u$. Suppose then that $z_{1}$ is not adjacent to $u$, and thus $z_{1}$ is a neighbor of a vertex $z_{1}^{\prime} \in C$ such that $u v z_{1}^{\prime}$ is a face. But then $u, v$ and $z_{1}$ have a common neighbor in $C$, and thus $r \neq z_{1}$. By a symmetric argument, $r \neq z_{2}$.

Since $u \prec r$, the vertex $r$ is not a friend of $u$, and thus $u$ has at most $a(v)-4$ friends via $v$. 
Lemma 9. If $(G, K, C)$ is a minimal counterexample, then $G$ contains no path $P=v_{1} v_{2} \ldots v_{k}$ with $k \geq 2$ disjoint from $K$, such that $v_{1}$ is a $(5, \leq 1)$ vertex, $v_{2}, \ldots, v_{k-1}$ are $(6,0)$-vertices, and $v_{k}$ is a $(5, \leq 2)$-vertex.

Proof. Suppose for a contradiction that $G$ contains such a path $P$. Without loss of generality, $P$ is an induced path. Furthermore, $P$ is disjoint from $C$ by Lemma 3 . Note that each vertex of $P$ has at most 4 neighbors in $V(G) \backslash(C \cup V(P))$, and thus $(G, K, C \cup V(P))$ is a target smaller than $(G, K, C)$. Let $\prec$ be a valid ordering of $(G, K, C \cup V(P))$, and let us extend the ordering to $(G, K, C)$ by setting $u \prec v_{1} \prec v_{2} \prec \ldots \prec v_{k}$ for every $u \in V(G) \backslash(C \cup V(P))$. Observe that $L_{G, C \cup V(P), \prec}(u)=L_{G, C, \prec}(u)$ for every $u \in V(G) \backslash(C \cup V(P))$. By Lemma 8, $v_{k}$ has at most one friend via each of its neighbors, and thus $\left|L_{G, C, \prec}\left(v_{k}\right)\right| \leq 7$. The vertex $v_{k-1}$ has at most 2 friends via $v_{k}$ and at most one friend via each of its neighbors distinct from $v_{k}$, and thus $\left|L_{G, C, \prec}\left(v_{k-1}\right)\right| \leq 7$. Consider any $i=1, \ldots, k-2$. By Lemma 8 , the vertex $v_{i}$ has at most 2 friends via $v_{i+1}$ (because $v_{i+1}$ is a $(6,0)$-vertex and we can set $\left.r=v_{i+2}\right)$ and at most one friend via each of its neighbors distinct from $v_{i+1}$, and thus $\left|L_{G, C, \prec}\left(v_{i}\right)\right| \leq 7$. Therefore, $\prec$ is a valid ordering for $(G, K, C)$, which is a contradiction.

Lemma 10. If $(G, K, C)$ is a minimal counterexample, then $G$ contains no induced cycle $Q=v_{1} v_{2} \ldots v_{k}$ with $k \geq 4$ disjoint from $K$, such that $v_{k}$ is a $(5, \leq 2)$-vertex and $v_{1}, \ldots, v_{k-1}$ are $(6,0)$-vertices.

Proof. Suppose for a contradiction that $G$ contains such an induced cycle $Q$. Clearly, $Q$ is disjoint from $C$ by Lemma 3. Note that each vertex of $Q$ has at most 4 neighbors in $V(G) \backslash(C \cup V(Q))$, and thus $(G, K, C \cup V(Q))$ is a target smaller than $(G, K, C)$. Let $\prec$ be a valid ordering of $(G, K, C \cup V(Q))$, and let us extend the ordering to $(G, K, C)$ by setting $u \prec v_{1} \prec v_{2} \prec \ldots \prec v_{k}$ for every $u \in V(G) \backslash(C \cup V(Q))$. Observe that $L_{G, C \cup V(Q), \prec}(u)=L_{G, C, \prec}(u)$ for every $u \in V(G) \backslash(C \cup V(Q))$. By Lemma 8 , $v_{k}$ has at most one friend via each of its neighbors, and thus $\left|L_{G, C, \prec}\left(v_{k}\right)\right| \leq 7$. The vertex $v_{k-1}$ has at most 2 friends via $v_{k}$ and at most one friend via each of its neighbors distinct from $v_{k}$, and thus $\left|L_{G, C, \prec}\left(v_{k-1}\right)\right| \leq 7$. Consider any $i=2, \ldots, k-2$. By Lemma 8 , the vertex $v_{i}$ has at most 2 friends via $v_{i+1}$ (because $v_{i+1}$ is a $(6,0)$-vertex and we can set $\left.r=v_{i+2}\right)$ and at most one friend via each of its neighbors distinct from $v_{i+1}$, and thus $\left|L_{G, C, \prec}\left(v_{i}\right)\right| \leq 7$. Finally, the $(6,0)$-vertex $v_{1}$ has at most two friends via $v_{2}$, at most one friend via $v_{k}$ (since we can set $r=v_{k-1}$ ), and at most one friend via each of its neighbors distinct from $v_{2}$ and $v_{k}$, and thus $\left|L_{G, C, \prec}\left(v_{1}\right)\right| \leq 7$. Therefore, $\prec$ is a valid ordering for $(G, K, C)$, which is a contradiction. 
Lemma 11. If $(G, K, C)$ is a minimal counterexample, then $G$ contains no path $P=v_{1} v_{2} \ldots v_{k}$ with $k \geq 3$ disjoint from $K$, such that $v_{1}$ is a $(5, \leq 1)$ vertex, $v_{2}, \ldots, v_{k-2}$ are $(6,0)$-vertices (if $\left.k \geq 4\right), v_{k-1}$ is a $(6,1)$-vertex and $v_{k}$ is a $(5,0)$-vertex.

Proof. Suppose for a contradiction that $G$ contains such a path $P$. Without loss of generality, $P$ is an induced path $\left(v_{k}\right.$ has no neighbors in $P$ distinct from $v_{k-1}$ by Lemma 9 ). Note that $P$ is disjoint from $C$, and each vertex of $P$ has at most 4 neighbors in $V(G) \backslash(C \cup V(P))$, and thus $(G, K, C \cup$ $V(P))$ is a target smaller than $(G, K, C)$. Let $\prec$ be a valid ordering of $(G, K, C \cup V(P))$, and let us extend the ordering to $(G, K, C)$ by setting $u \prec v_{1} \prec v_{2} \prec \ldots \prec v_{k-2} \prec v_{k} \prec v_{k-1}$ for every $u \in V(G) \backslash(C \cup V(P))$. Observe that $L_{G, C \cup V(P), \prec}(u)=L_{G, C, \prec}(u)$ for every $u \in V(G) \backslash(C \cup V(P))$. By Lemma $8, v_{k-1}$ has at most one friend via each of its neighbors, and thus $\left|L_{G, C, \prec}\left(v_{k-1}\right)\right| \leq 7$. The vertex $v_{k}$ has at most 3 friends via $v_{k-1}$ and at most one friend via each of its neighbors distinct from $v_{k-1}$, and thus $\left|L_{G, C, \prec}\left(v_{k}\right)\right| \leq 7$. Consider any $i=1, \ldots, k-2$. By Lemma 8 , the vertex $v_{i}$ has at most 2 friends via $v_{i+1}$ (because we can set $r=v_{i+2}$ and either $v_{i+1}$ is a $(6,0)$-vertex, or $r$ is a $(5,0)$-vertex) and at most one friend via each of its neighbors distinct from $v_{i+1}$, and thus $\left|L_{G, C, \prec}\left(v_{i}\right)\right| \leq 7$. Therefore, $\prec$ is a valid ordering for $(G, K, C)$, which is a contradiction.

\section{Discharging}

Let us now proceed with the discharging phase of the proof. Let $(G, K, C)$ be a minimal counterexample. Let us assign charge $c_{0}^{\prime}(v)=10 \operatorname{deg}(v)-60$ to each vertex $v \in V(G)$. Since all faces of $G$ except possibly for the outer one have length three, we have $|E(G)|=3|V(G)|-3-|K|$, and thus

$$
\begin{aligned}
\sum_{v \in V(G)} c_{0}^{\prime}(v) & =-60|V(G)|+10 \sum_{v \in V(G)} \operatorname{deg}(v) \\
& =-60|V(G)|+20|E(G)|=-60-20|K| .
\end{aligned}
$$

Next, every vertex of $v \in V(G) \backslash C$ sends charge of 5 to every adjacent vertex in $C$, and let $c_{0}$ denote the resulting assignment of charge. Since the total amount of charge does not change, we have $\sum_{v \in V(G)} c_{0}(v)=-60-20|K|$. If $v \in C$, then $\operatorname{deg}(v)=4, c_{0}^{\prime}(v)=-20$, and $v$ receives 5 from each of its neighbors, and thus $c_{0}(v)=0$. An $(a, b)$-vertex $v \in V(G) \backslash C$ has $c_{0}^{\prime}(v)=10 a+10 b-60$ and $v$ sends 5 to $b$ of its neighbors, and thus $c_{0}(v)=$ $10 a+5 b-60$. 
We say that a vertex $v \in V(G) \backslash C$ is big if $v \in K$ or $c_{0}(v)>0$ (i.e., $v$ is not a $(4,4)$-vertex, a $(5, \leq 2)$-vertex, or a $(6,0)$-vertex $)$. We call vertices not belonging to $K$ internal. Next, we redistribute the charge according to the following rules, obtaining the final charge $c$.

R1 Every big vertex sends 2 to each neighboring internal $(5,0)$-vertex.

R2 Every big vertex sends 1 to each neighboring internal $(5,1)$-vertex.

R3 If $v_{1} v_{2} \ldots v_{k}$ with $k \geq 3$ is a path in $G$ such that $v_{1} x v_{2}, v_{2} x v_{3}, \ldots$, $v_{k-1} x v_{k}$ are faces for some vertex $x, v_{1}$ is big, $x$ is either big or an internal $(6,0)$-vertex, $v_{2}, \ldots, v_{k-1}$ are internal $(6,0)$-vertices, and $v_{k}$ is an internal $(5, \leq 1)$-vertex, then $v_{1}$ sends 1 to $v_{k}$.

In the case of rule R3, we say that the charge arrives to $v_{k}$ through pair $\left(v_{k-1}, x\right)$, and departs $v_{1}$ through pair $\left(v_{2}, x\right)$. Note that it is possible for charge to arrive through $\left(x, v_{k-1}\right)$ or depart through $\left(x, v_{2}\right)$ as well, if $x$ is an internal $(6,0)$-vertex. If the charge departs through both $\left(v_{2}, x\right)$ and $\left(x, v_{2}\right)$, we say that the edge $v_{2} x$ is heavy for $v_{1}$. The key observations concerning the rule R3 are the following.

Lemma 12. Let $(G, K, C)$ be a minimal counterexample, let $v$ be an internal $(5, \leq 1)$-vertex, and let $v u_{1} x$ be a face of $G$. If $u_{1}$ is an internal $(6,0)$-vertex, then charge arrives to $v$ through $\left(u_{1}, x\right)$.

Proof. By Lemma 9, $x$ is not an internal $(5, \leq 2)$-vertex, and by Corollary 4 , $x$ is not a $(4,4)$-vertex. Hence, $x$ is either big or an internal $(6,0)$-vertex.

Let $v u_{1} x, u_{1} u_{2} x, u_{2} u_{3} x, \ldots, u_{k-1} u_{k} x$ be faces of $G$ incident with $x$ in order, where $k \geq 2$ is chosen minimum such that $u_{k}$ is not an internal $(6,0)$ vertex (possibly $u_{k}=v$ ). If $u_{k}$ is big, then it sends charge to $v$ by R3 and this charge arrives through $\left(u_{1}, x\right)$. Hence, assume that $u_{k}$ is not big. Since $u_{k-1}$ is a $(6,0)$-vertex, Corollary 4 implies that $u_{k}$ is not a $(4,4)$-vertex. Therefore, $u_{k}$ is an internal $(5, \leq 2)$-vertex. By Lemma 9, it follows that $u_{k}=v$. Since $x$ does not have a big neighbor, $x$ is an internal vertex. Since $x$ is internal big or $(6,0)$-vertex, its degree is at least 6 , and thus $k \geq 6$. However, Lemma 6 implies that $v u_{1} u_{2} \ldots u_{k-1}$ is an induced cycle, which contradicts Lemma 10 .

Lemma 13. Let $(G, K, C)$ be a minimal counterexample, let $v$ be a big vertex, and let $v u_{1} u_{2}, v u_{2} u_{3}$, and $v u_{3} u_{4}$ be distinct faces of $G$.

- If $u_{1} u_{2}$ is heavy for $v$, and $u_{1} u_{2} w$ is the face of $G$ with $w \neq v$, then $w$ is an internal $(5, \leq 1)$-vertex. Furthermore, no charge departs $v$ through $\left(u_{2}, u_{3}\right)$, and $u_{3} u_{4}$ is not heavy for $v$. 
- If $u_{1}$ is an internal $(5, \leq 1)$-vertex, then charge does not depart $v$ through $\left(u_{2}, u_{3}\right)$.

- If $v$ is an internal $(6,1)$-vertex adjacent to an internal $(5,0)$-vertex and charge departs $v$ through $\left(u_{1}, u_{2}\right)$, then $u_{3}$ is an internal $(5,0)$-vertex.

Proof. Suppose that charge departs $v$ through both $\left(u_{1}, u_{2}\right)$ and $\left(u_{2}, u_{1}\right)$. By the assumptions of the rule R3, both $u_{1}$ and $u_{2}$ are internal $(6,0)$-vertices. For $i=1,2$, there exists a path starting in $u_{i}$, passing through internal $(6,0)$-vertices adjacent to $u_{3-i}$, and ending in an internal $(5, \leq 1)$-vertex $x_{i}$ adjacent to $u_{3-i}$. By Lemma 9, we have $x_{1}=x_{2}$. Hence, $u_{1} u_{2} x_{1}$ is a triangle, and by Lemma 6, we have $w=x_{1}=x_{2}$.

- Suppose that in this situation, charge departs through $\left(u_{2}, u_{3}\right)$ because of a path in the neighborhood of $u_{3}$ ending in an internal $(5, \leq 1)$-vertex $x$. By Lemma 9, we have $x=w$, and by Lemma 6, $u_{2} u_{3} w$ bounds a face. However, then $u_{2}$ has degree 4 , which is a contradiction since $u_{2}$ is a $(6,0)$-vertex.

- Suppose that in this situation, $u_{3} u_{4}$ is heavy for $v$. Then the vertex $w^{\prime} \neq v$ of the face $u_{3} u_{4} w^{\prime}$ is an internal $(5, \leq 1)$-vertex, and by Lemma 9, we have $w=w^{\prime}$. By Lemma 6, it follows that $u_{2}$ and $u_{3}$ have degree 4 , which is a contradiction, since they are $(6,0)$-vertices.

Suppose now that $u_{1}$ is an internal $(5, \leq 1)$-vertex, and that charge departs $v$ through $\left(u_{2}, u_{3}\right)$ because of a path in the neighborhood of $u_{3}$ ending in an internal $(5, \leq 1)$-vertex $x$. By Lemma 9, we have $x=u_{1}$. But then $u_{3}$ is adjacent to $x$, and Lemma 6 would imply that $u_{1} u_{2} u_{3}$ is a face and $u_{2}$ has degree three, which is a contradiction.

Suppose that $v$ is an internal $(6,1)$-vertex adjacent to an internal $(5,0)$ vertex $z$ and that charge departs $v$ through $\left(u_{1}, u_{2}\right)$ because of a path in the neighborhood of $u_{2}$ ending in an internal $(5, \leq 1)$-vertex $x$. By Lemma 11 , we have $x=z$. But then $u_{2}$ is adjacent to $z$, and the triangle $u_{2} v z$ bounds a face by Lemma 6. Hence, $z=u_{3}$.

Let us now analyze the final charge of the vertices of $G$.

Lemma 14. Let $(G, K, C)$ be a minimal counterexample. If $v$ is an internal $(5,0)$-vertex of $G$, then $c(v) \geq 0$.

Proof. We have $c_{0}(v)=-10$.

By Corollary 4 and Lemma 9, every neighbor of $v$ in $G$ is either big or an internal $(6,0)$-vertex. Suppose that $v$ is adjacent to $\beta$ big vertices; each 
of them sends 2 to $v$ by the rule R1. By Lemma 12, charge arrives to $v$ through $10-2 \beta$ pairs. Hence, $c(v)=c_{0}(v)+2 \beta+(10-2 \beta)=0$.

Lemma 15. Let $(G, K, C)$ be a minimal counterexample. If $v$ is an internal $(5,1)$-vertex of $G$, then $c(v) \geq 0$.

Proof. We have $c_{0}(v)=-5$.

By Corollary 4 and Lemma 9, all neighbors of $v$ except for the one belonging to $C$ are either big or internal $(6,0)$-vertices. Let $v_{1}, \ldots, v_{6}$ be the neighbors of $v$ in order, where $v_{2} \in C$. Since $(6,0)$-vertices have no neighbor in $C$, both $v_{1}$ and $v_{3}$ are big. Let $\beta \geq 2$ be the number of big vertices adjacent to $v$; each of them sends 1 to $v$ by the rule R2. By Lemma 12, charge arrives to $v$ through $10-2 \beta$ pairs. Since $\beta \leq 5, c(v)=c_{0}(v)+\beta+(10-2 \beta) \geq 0$.

Lemma 16. Let $(G, K, C)$ be a minimal counterexample. If $v$ is a big $(a, b)$ vertex, then $c(v) \geq 8 a+7 b-60$. In particular, if $v$ is internal and $v$ is neither $a(6,1)$-vertex nor a $(7,0)$-vertex, then $c(v) \geq 0$.

Proof. By Lemma 6, the neighborhood of $v$ in $G$ induces a cycle, which we denote by $Q$. If $v$ is an internal vertex or $|K|=3$, then the length of $Q$ is $a+b$. If $v \in K$ and $|K|=2$ then the length of $Q$ is $a+b-1$. Note that if $v \in K$, then $a+b \geq 4$ by Corollary 7 .

Let us define a weight $w(e)$ for an edge $e=x y$ of $Q$ as follows. If charge departs $v$ through at least one of $(x, y)$ and $(y, x)$, then let $w(e)=2$. If $x$ or $y$ is an internal $(5, \leq 1)$-vertex and neither $x$ nor $y$ belongs to $C$, then let $w(e)=1$. Otherwise, let $w(e)=0$. Note that no two internal $(5, \leq 1)$ vertices are adjacent by Lemma 9, and that if charge departs $v$ through at least one of $(x, y)$ and $(y, x)$, then neither $x$ nor $y$ is an internal $(5, \leq 1)$ vertex. Furthermore, if $x y z$ is subpath of $Q$ and $y$ is an internal $(5,0)$-vertex, then $w(x y)=w(y z)=1$. We conclude that $\sum_{e \in E(Q)} w(e)$ is an upper bound on the amount of charge sent by $v$.

Note that $w(e) \leq 2$ for every $e \in E(Q)$, and $w(e)=0$ if $e$ is incident with a vertex of $C$. Since $C$ is an independent set, exactly $2 b$ edges of $Q$ are incident with a vertex of $C$, and thus $\sum_{e \in E(Q)} w(e) \leq 2(a+b-2 b)=2(a-b)$. Therefore, $c(v) \geq c_{0}(v)-2(a-b)=(10 a+5 b-60)-2(a-b)=8 a+7 b-60$.

If $a \geq 8$, then $c(v) \geq 8 a-60 \geq 4$. If $a=7$ and $b \geq 1$, then $c(v) \geq$ $8 \cdot 7+7-60=3$. If $a=6$ and $b \geq 2$, then $c(v) \geq 8 \cdot 6+7 \cdot 2-60=2$. Finally, if $a=5$ and $b \geq 3$, then $c(v) \geq 8 \cdot 5+7 \cdot 3-60=1$. Hence, if $v$ is an internal big vertex, it follows that $c(v) \geq 0$ unless $a=7$ and $b=0$, or $a=6$ and $b=1$. 
Lemma 17. Let $(G, K, C)$ be a minimal counterexample. If $v$ is an internal $(7,0)$-vertex, then $c(v) \geq 0$.

Proof. Note that $c_{0}(v)=10$. Let $v_{1} v_{2} \ldots v_{7}$ denote the cycle induced by the neighbors of $v$, and let $n_{5}$ denote the number of internal $(5, \leq 1)$-vertices of $G$ adjacent to $v$.

Since no two internal $(5, \leq 1)$-vertices are adjacent, it follows that $n_{5} \leq$ 3. By rules R1 and R2, the vertex $v$ sends at most $2 n_{5}$ units of charge. Furthermore, $v$ sends charge over at most $7-2 n_{5}$ edges by rule R3. If $n_{5} \geq 2$, then $c(v) \geq c_{0}(v)-2 n_{5}-2\left(7-2 n_{5}\right)=2\left(n_{5}-2\right) \geq 0$.

If $n_{5}=1$, then suppose that $v_{1}$ is the internal $(5, \leq 1)$-vertex. By Lemma 13, no charge departs $v$ through $\left(v_{2}, v_{3}\right)$ or $\left(v_{7}, v_{6}\right)$. Also, at most one of the edges $v_{3} v_{4}, v_{4} v_{5}$, and $v_{5} v_{6}$ is heavy for $v$. Therefore, charge departs $v$ through at most 6 pairs, and $c(v) \geq c_{0}(v)-2 n_{5}-6>0$.

Finally, suppose that $n_{5}=0$. By Lemma 13, for $i=1, \ldots, 7$, at most one of the edges $v_{i} v_{i+1}, v_{i+1} v_{i+2}, v_{i+2} v_{i+3}$ (with indices taken cyclically) is heavy. Therefore, charge departs $v$ through at most 9 pairs. Hence, $c(v) \geq c_{0}(v)-9>0$.

Lemma 18. Let $(G, K, C)$ be a minimal counterexample. If $v$ is an internal $(6,1)$-vertex, then $c(v) \geq 0$.

Proof. Note that $c_{0}(v)=5$. Let $Q=v_{1} v_{2} \ldots v_{7}$ denote the cycle induced by the neighbors of $v$, where $v_{2} \in C$.

Suppose first that $v$ is adjacent to an internal $(5,0)$-vertex, to which $v$ sends 2 by the rule R1. By Lemma 11, $v$ is adjacent only to one internal $(5,0)$-vertex and no other internal $(5, \leq 1)$-vertex. Furthermore, by the third part of Lemma 13, charge departs $v$ through at most two pairs. Hence, $c(v) \geq c_{0}(v)-2-2>0$.

Hence, we can assume that $v$ is not adjacent to internal $(5,0)$-vertices. Let $n_{5}$ be the number of internal $(5,1)$-vertices adjacent to $v$; $v$ sends 1 to each of them by the rule R2. Note that $n_{5} \leq 3$, since no two internal $(5,1)$-vertices are adjacent by Lemma 9. Since $v_{2} \in C$, neither $v_{1}$ nor $v_{3}$ is a $(6,0)$-vertex, and thus the edges $v_{1} v_{7}$ and $v_{3} v_{4}$ are not heavy for $v$.

Suppose first that $n_{5}=0$. If no edge of $Q$ is heavy for $v$, then charge departs $v$ through at most 5 pairs and $c(v) \geq c_{0}(v)-5=0$. Hence, by symmetry we can assume that $v_{4} v_{5}$ or $v_{5} v_{6}$ is heavy for $v$. Lemma 13 implies that no other edge of $Q$ is heavy for $v$. Let us distinguish the cases.

- If $v_{4} v_{5}$ is heavy, then Lemma 13 implies that charge does not depart through the pair $\left(v_{4}, v_{3}\right)$, and it does not depart through the pair $\left(v_{3}, v_{4}\right)$ since $v_{3}$ is not a $(6,0)$-vertex. 
- If $v_{5} v_{6}$ is heavy, then Lemma 13 implies that the common neighbor $w \neq v$ of $v_{5}$ and $v_{6}$ is an internal $(5, \leq 1)$-vertex, and furthermore, that charge may only depart $v$ through pairs $\left(v_{4}, v_{5}\right),\left(v_{7}, v_{6}\right),\left(v_{4}, v_{3}\right)$, and $\left(v_{7}, v_{1}\right)$ in addition to $\left(v_{5}, v_{6}\right)$ and $\left(v_{6}, v_{5}\right)$.

Suppose that the charge departs $v$ through all these pairs. By Lemma9, all the charge arrives to $w$. However, then $w$ is adjacent to $v_{1}, v_{3}, v_{5}$, $v_{6}$, as well as at least two $(6,0)$-vertices of the paths showing that the charge departing through the pairs $\left(v_{4}, v_{5}\right)$ and $\left(v_{7}, v_{6}\right)$ arrives to $w$. This is a contradiction, since $w$ has at most 5 neighbors not belonging to $C$.

In both cases, we conclude that charge departs $v$ through at most 5 pairs, and thus $c(v) \geq c_{0}(v)-5=0$.

Suppose now that $n_{5}=1$. If neither $v_{1}$ nor $v_{3}$ is an internal $(5,1)$-vertex, then $v$ sends charge over at most three edges by the rule $\mathrm{R} 3$ and at most one of them is heavy for $v$ by Lemma 13, and $c(v) \geq c_{0}(v)-n_{5}-4=0$. Hence, by symmetry, we can assume that $v_{3}$ is an internal $(5,1)$-vertex. By Lemma 13. only one of the edges $v_{5} v_{6}$ and $v_{6} v_{7}$ may be heavy. If $v_{6} v_{7}$ is heavy, then charge does not depart $v$ through $\left(v_{7}, v_{1}\right)$ or $\left(v_{1}, v_{7}\right)$, by Lemma 13 and since $v_{1}$ is not a $(6,0)$-vertex. If $v_{5} v_{6}$ is heavy, then charge does not depart $v$ through $\left(v_{4}, v_{5}\right)$ or $\left(v_{5}, v_{4}\right)$ by Lemma 13. In either case, charge departs $v$ through at most 4 pairs, and again $c(v) \geq 0$.

Suppose that $n_{5}=2$. Recall that no two internal $(5, \leq 1)$-vertices are adjacent by Lemma 9. If at least one of $v_{1}$ and $v_{3}$ is not an internal $(5,1)$ vertex, then $v$ sends charge over at most two edges by rule $\mathrm{R} 3$ and neither of them is heavy for $v$ by Lemma 13, hence $c(v) \geq c_{0}(v)-n_{5}-2>0$. If both $v_{1}$ and $v_{3}$ are internal $(5,1)$-vertices, then only the edge $v_{5} v_{6}$ may be heavy for $v$ by Lemma 13, and if it is heavy, then no charge departs $v$ through $\left(v_{4}, v_{5}\right),\left(v_{5}, v_{4}\right),\left(v_{6}, v_{7}\right)$ and $\left(v_{7}, v_{6}\right)$. Hence, charge departs $v$ through at most 3 pairs and $c(v) \geq c_{0}(v)-n_{5}-3=0$.

Finally, suppose that $n_{5}=3$. In this case, Lemma 13 shows that no charge departs $v$, and thus $c(v)=c_{0}(v)-n_{5}>0$.

Proof of Theorem 2. Suppose for a contradiction that Theorem 2 is false. Then, there exists a minimal counterexample $(G, K, C)$. Assign and redistribute charge among its vertices as we described above. Note that the redistribution of the charge does not change its total amount, and thus

$$
\sum_{v \in V(G)} c(v)=\sum_{v \in V(G)} c_{0}(v)=-60-20|K|
$$


Recall that $c(v)=c_{0}(v)=0$ for every $v \in C$. If $v$ is an internal big vertex, then $c(v) \geq 0$ by Lemmas 16, 17 and 18. If $v$ is an internal vertex with $c_{0}(v)$ negative, then by Lemma 5, it follows that $v$ is either a $(5,0)$-vertex, or a $(5,1)$-vertex, and $c(v) \geq 0$ by Lemmas 14 and 15. If $v$ is an internal vertex with $c_{0}(v)=0$ (i.e., $v$ is a $(4,4)$-vertex, or a $(5,2)$-vertex, or a $(6,0)$-vertex), then $c(v)=c_{0}(v)=0$. Therefore,

$$
\sum_{v \in V(G)} c(v) \geq \sum_{v \in K} c(v)
$$

Consider an $(a, b)$-vertex $v \in K$. Since $v$ is incident with two edges of the outer face of $G$, we have $a \geq 2$, and $a+b \geq 4$ by Corollary 7 , By Lemma 16, $c(v) \geq 8 \cdot 2+7 \cdot 2-60=-30$. Therefore,

$$
\sum_{v \in K} c(v) \geq-30|K|
$$

However, since $|K| \leq 3$, we have $-30|K|>-60-20|K|$, which is a contradiction. Therefore, no counterexample to Theorem 2 exists.

\section{References}

[1] S. A. Burr AND P. ERDős, On the magnitude of generalized Ramsey numbers for graphs, in Infinite and finite sets (Colloq., Keszthely, 1973; dedicated to P. Erdős on his 60th birthday), Vol. 1, North-Holland, Amsterdam, 1975, pp. 215-240. Colloq. Math. Soc. János Bolyai, Vol. 10.

[2] G. Chen And R. Schelp, Graphs with linearly bounded Ramsey numbers, J. Combin. Theory, Ser. B, 57 (1993), pp. 138-149.

[3] H. Kierstead, B. Mohar, S. Špacapan, D. Yang, and X. Zhu, The two-coloring number and degenerate colorings of planar graphs, SIAM J. Discrete Math., 23 (2009), pp. 1548-1560.

[4] H. Kierstead And W. Trotter, Planar graph coloring with an uncooperative partner, J. Graph Theory, 18 (1994), pp. 569-584. 